\title{
PHASE TRANSITIONS IN NEUTRON STARS AND MAXIMUM MASSES
}

\author{
H. HEISELBERG \\ Nordita, Blegdamsvej 17, DK-2100 Copenhagen Ø, Denmark \\ and \\ M. HJORTH-JENSEN \\ Department of Physics, University of Oslo, N-0316 Oslo, Norway
}

\begin{abstract}
Using the most recent realistic effective interactions for nuclear matter with a smooth extrapolation to high densities including causality, we constrain the equation of state and calculate maximum masses of rotating neutron stars. First and second order phase transitions to, e.g., quark matter at high densities are included. If neutron star masses of $2.3 M_{\odot}$ from quasi-periodic oscillation in low mass X-ray binaries are confirmed, soft equation of state as well as strong phase transitions can be excluded in cores of neutron stars.
\end{abstract}

Subject headings: dense matter-stars:neutron

\section{INTRODUCTION}

The best determined neutron star masses are found in binary pulsars and all lie in the range 1.4$1.5 M_{\odot}$ (see Thorsett and Chakrabarty 1999) except for the nonrelativistic pulsar PSR J1012+5307 of mass $M=(2.1 \pm 0.4) M_{\odot}$ (Paradijs 1999). Two X-ray binary masses have been measured, Vela $\mathrm{X}-1$ with $M=(1.9 \pm 0.1) M_{\odot}$ and Cygnus X-2 with $M=(1.8 \pm 0.2) M_{\odot}$. The recent discovery of highfrequency brightness oscillations in low-mass X-ray binaries provides a new promising method for determining masses and radii of neutron stars (see Miller, Lamb, \& Psaltis 1998, Zhang, Strohmayer, \& Swank 1997, Kaaret, Ford, \& Chen 1997). These quasi-periodic oscillations (QPO) are most likely the orbital frequencies $\nu_{Q P O}=(1 / 2 \pi) \sqrt{G M / r^{3}}$ of accreting gas in Keplerian orbits of radius $r$ around neutron stars of mass $M$. According to Miller, Lamb, \& Psaltis (1999) the gas can be tracked to its innermost stable orbit, $R_{i m s}=6 G M / c^{2}$ for a slowly rotating star, whereby the neutron star mass can be deduced, $M \simeq 2.2 M_{\odot} \frac{1000 \mathrm{~Hz}}{\nu^{Q P O}}$. For example, the maximum frequency of $1200 \mathrm{~Hz}$ QPO observed in $4 \mathrm{U} 1820-30$ gives $M \simeq 2.3 M_{\odot}$. Such large masses severely restricts the EoS for dense matter as addressed in the following.

Recent models for the nucleon-nucleon interaction have reduced the uncertainty in the nuclear equation of state (EoS) allowing for more reliable calculations of neutron star properties, see 
Akmal, Pandharipande, \& Ravenhall (1998) and Engvik et al. (1997). Likewise, recent realistic effective interactions for nuclear matter obeying causality at high densities, constrain the equation of state severely and thus also the maximum masses of neutron stars, see Akmal, Pandharipande, \& Ravenhall (1998) and Kalogera \& Baym (1996). We will here elaborate on these analyses by incorporating causality smoothly in the EoS for nuclear matter and allow for first and second order phase transitions to, e.g., quark matter. Finally, results are compared with observational neutron star masses and concluding remarks are given.

\section{THE NUCLEAR EOS}

For the discussion of the gross properties of neutron stars we will use the EoS of Akmal, Pandharipande, \& Ravenhall (1998) (hereafter APR98) which is a non-relativistic EoS based on the most recent models for the nucleon-nucleon interaction, see Engvik et al. (1997) for a discussion of these models, and with the inclusion of a parametrized three-body force and relativistic boost corrections. The EoS for nuclear matter is thus known to some accuracy for densities up to a few times nuclear saturation density $n_{0}=0.16 \mathrm{fm}^{-3}$. We parametrize the APR98 EoS by a simple form that gives a good fit around nuclear saturation densities and smoothly incorporates causality at high densities. The parametrization consists of a compressional term and a symmetry energy term

$$
\mathcal{E}=E_{\text {comp }}(n)+S(n)(1-2 x)^{2}=\mathcal{E}_{0} u \frac{u-2-\delta}{1+\delta u}+S_{0} u^{\gamma}(1-2 x)^{2} .
$$

Here, $n=n_{p}+n_{n}$ is the total baryon density, $x=n_{p} / n$ the proton fraction and $u=n / n_{0}$ is the ratio of the baryon density to nuclear saturation density. The compressional term is in Eq. (1) parametrized by a simple form which reproduces the saturation density and the binding energy per nucleon $\mathcal{E}_{0}=15.8 \mathrm{MeV}$ at $n_{0}$ of APR98. The parameter $\delta \simeq 0.2$, which gave the best fit to the data of APR98 (see Heiselberg \& Hjorth-Jensen 1999) is determined by fitting the energy per nucleon of APR98 up to densities of $n \sim 4 n_{0}$. For the symmetry energy term we obtain $S_{0}=32 \mathrm{MeV}$ and $\gamma=0.6$ for the best fit. The proton fraction is given by $\beta$-equilibrium at a given density. Besides reproducing the properties of nuclear matter around $n_{0}$, the parametrization

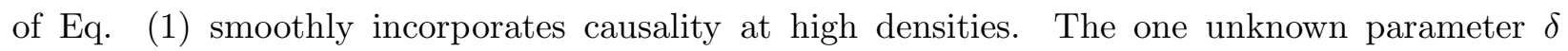
expresses the uncertainty in the EoS at high density and we shall vary this parameter within the allowed limits in the following with and without phase transitions to calculate mass, radius and density relations for neutron stars. The "softness" parameter $\delta$ is related to the incompressibility of nuclear matter as $K_{0}=18 \mathcal{E}_{0} /(1+\delta) \simeq 200 \mathrm{MeV}$. It agrees with the poorly known experimental value, $K_{0} \simeq 180-280 \mathrm{MeV}$ which does not restrict $\delta$ very well. From $\left(v_{s} / c\right)^{2}=\partial P / \partial(n \mathcal{E})$, where $P$ is the pressure, and the EoS of Eq. (11) we obtain that

$$
\delta \gtrsim \sqrt{\frac{\mathcal{E}_{0}}{m_{n}}} \simeq 0.13,
$$


i.e., for values of $\delta \gtrsim 0.13$ the EoS remains causal for all densities. With this condition we have an EoS that reproduces the data of APR98 at densities up to $0.6 \sim 0.7 \mathrm{fm}^{-3}$ and has the right causal behavior at higher densities. The EoS of APR98 becomes superluminal at $n \approx 1.1 \mathrm{fm}^{-3}$. For larger $\delta$ values the EoS is softer which eventually leads to smaller maximum masses of neutron star. The observed $M \simeq 1.5 M_{\odot}$ in binary pulsars restricts $\delta<0.4-0.5$ depending on rotation as shown in calculations of neutron stars below. Furthermore, for realistic values for the bag parameter and quark coupling constant $\alpha_{s}$, the mixed baryon-quark phase starts at densities around $0.5-0.8 \mathrm{fm}^{-3}$, and the differences at higher densities will not be of importance in our analysis of the dynamics and structure of neutrons stars.

In Fig. 1 we plot the sound speed $\left(v_{s} / c\right)^{2}$ for various $\delta$ values and that resulting from the microscopic calculation of APR98 for $\beta$-stable pn-matter. The form of (11), with the inclusion of the parameter $\delta$, provides therefore a smooth extrapolation from small and large densities with the correct behavior in both limits, i.e. the binding energy per nucleon $E / A=\mathcal{E}$ is linear in number density. In the dilute limit this is the Lenz (optical) potential. At high densities the linearity is required by the condition that the sound speed $v_{s}$ does not exceed the speed of light. This justifies the introduction of the parameter $\delta$ in our parametrization. For $\delta=0.1$ the EoS becomes superluminal at densities of the order of $6 \mathrm{fm}^{-3}$.

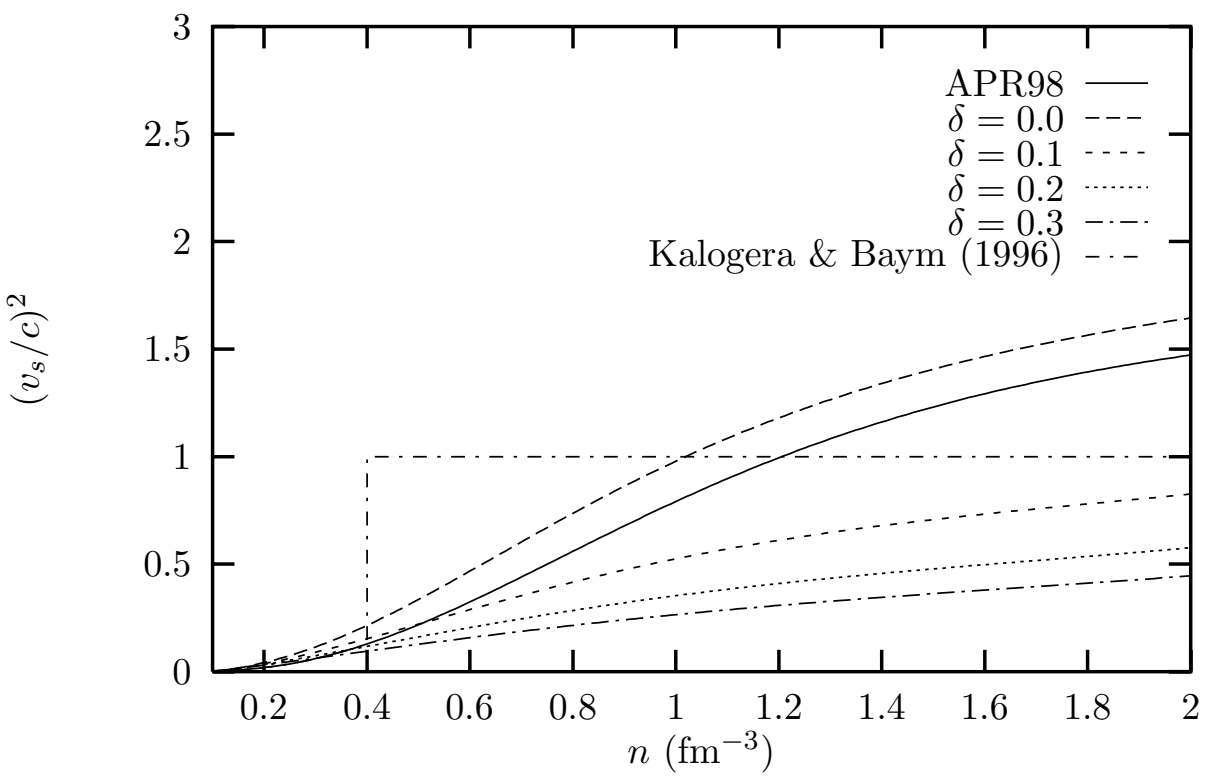

Fig. 1.- $\left(v_{s} / c\right)^{2}$ for $\beta$-stable $p n$-matter for $\delta=0,0.1,0.2,0.3$, the results of APR98, and for the patched EoS of Kalogera \& Baym (1996) which shows a discontinuous $\left(v_{s} / c\right)^{2}$.

The sound speed of Kalogera \& Baym (1996) is also plotted in Fig. 1. It jumps discontinuously to the speed of light at a chosen density in order to keep the EoS causal. With this prescription, 
Kalogera \& Baym (1996) were able to obtain an upper bound for neutron star masses of $2.9 M_{\odot}$. The approach of Baym and Kalogera differs substantially from ours since their EoS is discontinuously stiffened by taking $v_{s}=c$ at densities above a certain value $n_{c}$ which, however, is lower than $n_{s}=5 n_{0}$ where their nuclear EoS becomes superluminal. This stiffens the nuclear EoS for densities $n_{c}<n<n_{s}$ but softens it at higher densities. Their resulting maximum masses lie in the range $2.2 M_{\odot}<M<2.9 M_{\odot}$. Our approach incorporates causality by reducing the sound speed smoothly towards the speed of light at high densities. Therefore our maximum mass will never exceed that of the nuclear EoS of APR98. In fact one may argue that at very high densities particles become relativistic and the sound speed should be even lower, $v_{s}^{2} \simeq c^{2} / 3$. Therefore the softening we get from incorporating causality is even on the low side and the maximum masses are upper estimates.

\section{PHASE TRANSITIONS}

The physical state of matter in the interiors of neutron stars at densities above a few times normal nuclear matter densities is essentially unknown and many first and second order phase transitions have been speculated upon. We will specifically study the hadron to quark matter transition at high densities, but note that other transitions as, e.g., kaon and/or pion condensation or the presence of other baryons like hyperons also soften the EoS and thus further aggravate the resulting reduction in maximum masses.

Since we do not have a fully reliable theory for the quark matter phase, we will for simplicity employ the bag model in our actual studies of quark phases and neutron star properties. In the bag model the quarks in the hadrons are assumed to be confined to a finite region of space, the so-called 'bag', by a vacuum pressure $B$. The pressure from the quarks inside the bag is provided by the Fermi pressure and interactions computed to order $\alpha_{s}=g^{2} / 4 \pi$ where $g$ is the QCD coupling constant. The pressure for quarks of flavor $f$, with $f=u, d$ or $s$ is, see Kapusta (1988),

$$
\begin{aligned}
P_{f} & =\frac{1}{4 \pi^{2}}\left[\mu_{f} k_{f}\left(\mu_{f}^{2}-2.5 m_{f}^{2}\right)+1.5 m_{f}^{4} \ln \left(\frac{\mu_{f}+k_{f}}{m_{f}}\right)\right] \\
& -\frac{\alpha_{s}}{\pi^{3}}\left[\frac{3}{2}\left(\mu_{f} k_{f}-m_{f}^{2} \ln \left(\frac{\mu_{f}+k_{f}}{m_{f}}\right)\right)^{2}-k_{f}^{4}\right] .
\end{aligned}
$$

The Fermi momentum is $k_{f}=\left(\mu_{f}^{2}-m_{f}^{2}\right)^{1 / 2}$. The total pressure, including the bag constant $\mathrm{B}$ simulating confinement, is

$$
P=\sum_{f} P_{f}-B+P_{e}+P_{\mu}
$$

where $P_{e, \mu}$ are the electron and muon pressure, e.g., $P_{e}=\mu_{e}^{4} / 12 \pi^{2}$. A Fermi gas of quarks of flavor $i$ has density $n_{i}=k_{F i}^{3} / \pi^{2}$, due to the three color states. $\alpha$ is the strong interaction coupling constant, assumed to have a value of 0.5 . The $u$ and $d$ quarks are taken to be massless, and $s$ quarks to have a mass of $150 \mathrm{MeV}$. Typical quark chemical potentials $\mu_{q} \gtrsim m_{N} / 3$ are generally much larger. The 
value of the bag constant $B$ is poorly known, and we present results using two representative values, $B=150 \mathrm{MeVfm}^{-3}$ (Cleymans, Gavai \& Suhonen 1986) and $B=200 \mathrm{MeVfm}^{-3}$ (Satz 1982).

The quark and nuclear matter mixed phase described in Glendenning (1992) has continuous pressures and densities due to the general Gibbs criteria for two-component systems. There are no first order phase transitions but at most two second order phase transitions. Namely, at a lower density, where quark matter first appears in nuclear matter, and at a very high density (if gravitationally stable), where all nucleons are finally dissolved into quark matter. This mixed phase does, however, not include local surface and Coulomb energies of the quark and nuclear matter structures. If the interface tension between quark and nuclear matter is too large, the mixed phase is not favored energetically due to surface and Coulomb energies associated with forming these structures, see e.g., Heiselberg, Pethick, \& Staubo (1993) and Heiselberg \& HjorthJensen (1999). The neutron star will then have a core of pure quark matter with a mantle of nuclear matter surrounding it and the two phases are coexisting by a first order phase transition or Maxwell construction, see Fig. 2. For a small or moderate interface tension the quarks are confined in droplet, rod- and plate-like structures (Heiselberg, Pethick, \& Staubo 1993 and Heiselberg \& Hjorth-Jensen 1999) as found in the inner crust of neutron stars, see Lorenz, Ravenhall, \& Pethick (1993).

\section{NEUTRON STAR PROPERTIES}

In order to obtain the mass and radius of a neutron star, we have solved the Tolman-OppenheimerVolkov equation with and without rotational corrections following the approach of Hartle (1967). The equations of state employed are given by the $p n$-matter EoS with $\delta=0.13,0.2,0.3,0.4$ with nucleonic degrees of freedon only. In addition we have selected two representative values for the Bag-model parameter $B$, namely 150 and $200 \mathrm{MeVfm}^{-3}$ for our discussion on eventual phase transitions. The quark phase is linked with our pn-matter EoS from Eq. (11) with $\delta=0.2$ through either a mixed phase construction or a Maxwell construction, see Heiselberg and Hjorth-Jensen (1999) for further details. For $B=150 \mathrm{MeVfm}^{-3}$, the mixed phase begins at $0.51 \mathrm{fm}^{-3}$ and the pure quark matter phase begins at $1.89 \mathrm{fm}^{-3}$. Finally, for $B=200 \mathrm{MeVfm}^{-3}$, the mixed phase starts at 0.72 $\mathrm{fm}^{-3}$ while the pure quark phase starts at $2.11 \mathrm{fm}^{-3}$. In case of a Maxwell construction, in order to link the $p n$ and the quark matter EoS, we obtain for $B=150 \mathrm{MeVfm}^{-3}$ that the pure $p n$ phase ends at $0.92 \mathrm{fm}^{-3}$ and that the pure quark phase starts at $1.215 \mathrm{fm}^{-3}$, while the corresponding numbers for $B=200 \mathrm{MeVfm}^{-3}$ are 1.04 and $1.57 \mathrm{fm}^{-3}$.

As can be seen from Fig. 2 none of the equations of state from either the pure pn phase or with a mixed phase or Maxwell construction with quark degrees of freedom, result in stable configurations for densities above $\sim 10 n_{0}$, implying thereby that none of the stars have cores with a pure quark phase. The EoS with $p n$ degrees of freedom only results in the largest mass $\approx 2.2 M_{\odot}$ when rotational corrections are accounted for. With the inclusion of the mixed phase, the total mass is reduced since the EoS is softer. 

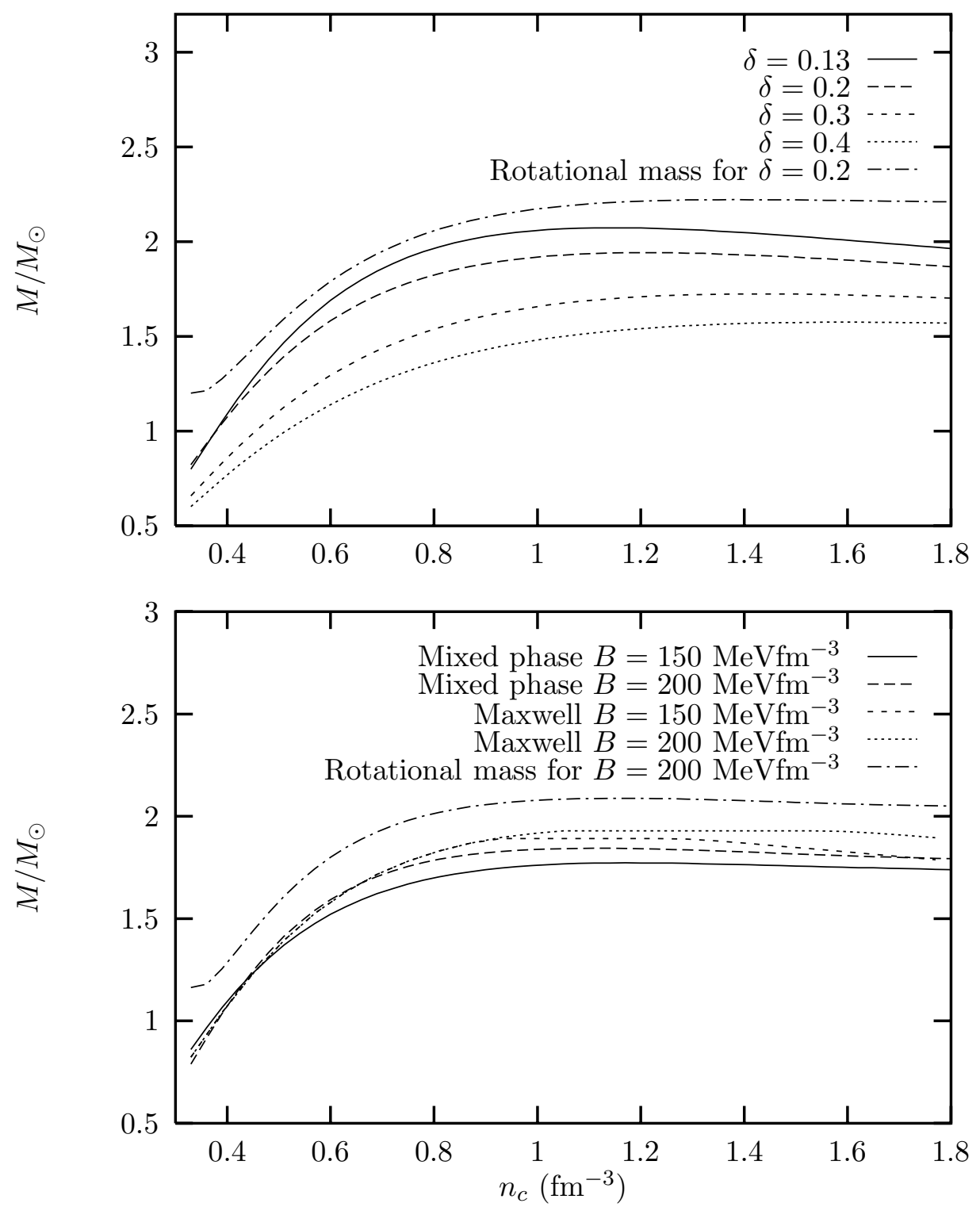

Fig. 2. - Total mass $M$ as function of central density $n_{c}$ for various values of $\delta$ (upper panel) and the bag parameter $B$ (lower panel) for both a mixed phase and a Maxwell contructed EoS with $\delta=0.2$ in Eq. 1). In addition we include also the rotational corrections for the pure $p n$-case with $\delta=0.2$ and the mixed phase contruction for $B=200 \mathrm{MeVfm}^{-3}$. For the Maxwell construction which exhibits a first order phase transition, in the density regions where the two phases coexist, the pressure is constant, a fact reflected in the constant value of the neutron star mass. All results are for $\beta$-stable matter. Note also that for the upper panel, the EoS for $\delta=0.3$ and $\delta=0.4$ start to differ from those with $\delta=0.13,0.2$ at densities below $0.2 \mathrm{fm}^{-3}$. 
Several interesting conclusions can be inferred from the results displayed in Fig. 2. Firstly, to obtain neutron star masses of the order $M \sim 2.2-2.3 M_{\odot}$ as may now have been observed in QPO's, we need the stiffest EoS allowed by causality, i.e. $\delta \simeq 0.13-0.2$, and to include rotation. The relation between the orbital frequency $\nu_{\mathrm{QPO}}$ and the mass of the star can, following Miller, Lamb \& Psaltis (1998), be approximated by

$$
\nu_{\mathrm{QPO}} \approx 2210(1+0.748 j) \frac{M_{\odot}}{M},
$$

with $j=c I \omega / M^{2}$ a dimensionless measure of the angular momentum of the star and with $\omega$ the stellar rotation. Inserting our results from Table 1 for the pure $p n$-matter EoS for $\delta=0.2$ gives $\nu_{\mathrm{QPO}}=1190 \mathrm{~Hz}$, in good agreement with the extracted value of Miller, Lamb \& Psaltis (1998) for the $1200 \mathrm{~Hz}$ QPO from $4 \mathrm{U}$ 1820-30. Secondly, a phase transition to quark matter below densities of order $\sim 5 n_{0}$ can be excluded, corresponding to restricting the Bag constant to $B>200 \mathrm{MeVfm}^{-3}$. This can be seen in Fig. 2 where we plot star masses as function of the central density $n_{c}$ and bag-model parameter $B$. These results differ significantly from those of APR98 and Kalogera \& Baym (1996) due to the very different recipes we use to incorporate causality at high densities. On the other hand, if it turns out that the QPOs are not from the innermost stable orbits and that even accreting neutron stars have small masses, say like the binary pulsars $M<1.5 M_{\odot}$, this may indicate that heavier neutron stars are not stable. Therefore, the EoS is soft at high densities $\delta>0.4$ or that a phase transition occurs at a few times nuclear matter densities. For the nuclear to quark matter transition this would require $B<80 \mathrm{MeVfm}^{-3}$ for $\delta=0.2$. For such small Bag parameters there is an appreciable quark and nuclear matter mixed phase in the neutron star interior but even in these extreme cases a pure quark matter core is not obtained for stable neutron star configurations.

\section{SUMMARY}

Modern NN-potentials have reduced the uncertainties in the calculated EoS. Using the most recent realistic effective interactions for nuclear matter of APR98 with a smooth extrapolation to high densities including causality, the equation of state could be constrained by a "softness" parameter $\delta$ which parametrizes the unknown stiffness of the EoS at high densities. Maximum masses were calculated for rotating neutron stars with and without first and second order phase transitions to, e.g., quark matter at high densities.

The comparison of calculated maximum masses to the observed ones leaves two natural options:

- Case I: The large masses of the neutron stars in $Q P O 4 U$ 1820-30 ( $\left.M=2.3 M_{\odot}\right)$, PSR $J 1012+5307\left(M=2.1 \pm 0.4 M_{\odot}\right)$, Vela $X-1\left(M=1.9 \pm 0.1 M_{\odot}\right)$, and Cygnus $X-2(M=$ $\left.1.8 \pm 0.2 M_{\odot}\right)$, are confirmed and complemented by other neutron stars with masses around $\sim 2 M_{\odot}$. 
Table 1: Maximum gravitational mass in $M_{\odot}$ and the corresponding radius $R$, in units of $\mathrm{km}$, for the given central density $n_{c}$, in units of $\mathrm{fm}^{-3}$, for various equations of state. The maximum moment of Inertia $I$, in units of $M_{\odot} \mathrm{km}^{2}$, is also listed. Note that this occurs for another central density than listed below. All results are for $\beta$-stable matter.

\begin{tabular}{ccccc}
\hline EoS & Max mass & Max $I$ & $R$ & $n_{c}$ \\
\hline$p n \delta=0.13$ & 2.07 & 110.1 & 11.0 & 1.1 \\
pn $\delta=0.2$ & 1.94 & 98.0 & 10.8 & 1.2 \\
pn $\delta=0.3$ & 1.72 & 78.7 & 10.3 & 1.4 \\
pn $\delta=0.4$ & 1.58 & 66.9 & 10.0 & 1.6 \\
\hline Mixed phase with $\delta=0.2$ & & & & \\
\hline$B=200 \mathrm{MeVfm}^{-3}$ & 1.84 & 91.5 & 11.6 & 1.1 \\
$B=150 \mathrm{MeVfm}^{-3}$ & 1.77 & 84.7 & 11.5 & 1.2 \\
\hline Rotational correction $\delta=0.2$ & & & & \\
\hline Mixed phase $B=200 \mathrm{MeVfm}^{-3}$ & 2.09 & 90.9 & 11.5 & 1.2 \\
pn-matter only & 2.22 & 94.6 & 10.5 & 1.4 \\
\hline
\end{tabular}


As a consequence, the EoS of dense nuclear matter is severely restricted and only the stiffest EoS allowed by causality are allowed, i.e., softness parameter $0.13 \leq \delta \lesssim 0.2$. Furthermore, any significant phase transition at densities below $<5 n_{0}$ can be excluded.

That the radio binary pulsars all have masses around $1.4 M_{\odot}$ is then probably due to the formation mechanism in supernovae and are related to the Chandrasekhar mass $M_{c h} \simeq 1.4 M_{\odot}$ of white dwarfs. Neutron stars can subsequently acquire larger masses by accretion as in X-ray binaries.

- Case II: The heavy neutron stars prove erroneous by more detailed observations and only the canonical $\sim 1.4 M_{\odot}$ masses are found.

If accretion does not produce neutron stars heavier than $\gtrsim 1.5 M_{\odot}$, this indicates that heavier neutron stars simply are not stable which in turn implies a soft EoS, either $\delta>0.4$ or a significant phase transition must occur already at a few times nuclear saturation densities.

Observations of neutron star masses as well radii, surface temperatures, etc., may resolve these questions in the near future.

Discussions with C.J. Pethick are gratefully acknowledged.

\section{REFERENCES}

Akmal, A., Pandharipande, V. R., \& Ravenhall, D. G., 1998, Phys. Rev. C, 58, 1804

Baym, G. A., \& Chin, S. A., 1976, Nucl. Phys. A, 262, 527.

Cleymans, J., Gavai, R.V., \& Suhonen, E., 1986, Phys. Rep., 130, 217

Engvik, L., Hjorth-Jensen, M., Machleidt, R., Müther, H., \& Polls A., 1997, Nucl. Phys. A, 627, 125

Glendenning, N., 1992, Phys. Rev. D, 46, 1274

Hartle, J. B., 1967, ApJ, 150, 1005

Heiselberg, H., \& Hjorth-Jensen, M., 1998, Phys. Rev. Lett., 80, 5485; and 1999, Phys. Rep., in press

Heiselberg, H., Pethick, C. J., \& Staubo, E. F., 1993, Phys. Rev. Lett., 70, 1355

Kaaret, P.,, Ford, E. C., \& Chen, K., 1997, ApJ, 480, L27

Kalogera, V., \& Baym, G., 1996, ApJ, 470, L61

Kapusta, J. I., 1989, Finite Temperature Field Theory, (Cambridge: Cambridge University Press) 
Miller, M. C., Lamb, F. K., \& Psaltis, P., 1998 ApJ, 508, 791.

Lorenz, C. P., Ravenhall, D. G., \& Pethick, C. J., 1993, Phys. Rev. Lett., 70, 379

van Paradijs, J., 1998, astro-ph/9802177 and in: The Many Faces of Neutron Stars, ed. R. Buccheri, J. van Paradijs \& M. A. Alpar, (Dordrecht: Kluwer), in press

Satz, H., 1982, Phys. Lett. B, 113, 245

Thorsett, S. E., \& Chakrabarty, D., 1999, ApJ, 512, 288

Zhang, W., Strohmayer, T. E., \& Swank, J. H., 1997, ApJ, 482, L167

This preprint was prepared with the AAS $\mathrm{LAT}_{\mathrm{E}} \mathrm{X}$ macros v4.0. 